International Journal of Pure and Applied Mathematics

Volume 85 No. 1 2013, 155-170

ISSN: 1311-8080 (printed version); ISSN: 1314-3395 (on-line version)

url: http://www.ijpam.eu

doi: http://dx.doi.org/10.12732/ijpam.v85i1.13

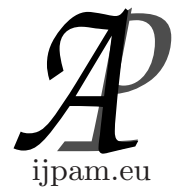

\title{
EXPLICIT CONDITIONS FOR EXISTING OF NON-OSCILLATING SOLUTIONS OF ODD ORDER LINEAR SYSTEM OF NEUTRAL TYPE WITH DISTRIBUTED DELAY
}

\author{
Atanaska Georgieva $^{1}$, Hristo Kiskinov $^{2}$, Andrey Zahariev ${ }^{3}$, Stoyan Zlatev ${ }^{4}$ \\ ${ }^{1,2,3,4}$ Faculty of Mathematics and Informatics \\ University of Plovdiv \\ 236, Bulgaria Blvd., Plovdiv, 4003, BULGARIA
}

\begin{abstract}
In the present paper we establish explicit and easy computable sufficient conditions for existing of bounded and unbounded non-oscillating solutions of linear delayed system of neutral type with distributed delay. The results are proved by logarithmic norm technique and are new even in the case of system with constant delays in neutral part. Moreover they are applicable in the case of non-monotone measures too.
\end{abstract}

AMS Subject Classification: $34 \mathrm{~K} 11,34 \mathrm{~K} 40,34 \mathrm{C} 10$

Key Words: neutral type, distributed delay, non-oscillatory solution, logarithmic norm, numerical range

\section{Introduction}

The neutral delay differential equations have applications in physics, biology and other real world life problems. For example the neutral equations appear in modelling of the networks containing lossless transmission lines (as in highspeed computers where the lossless transmission lines are used to interconnect switching circuits), in the study of vibrating masses attached to an elastic bar, in the theory of automatic control and in neuromechanical systems in which inertia plays an important role and etc. (see also $[13,14]$ ). The first detailed

Received: March 11, 2013

(C) 2013 Academic Publications, Ltd.

$\S$ Correspondence author url: www.acadpubl.eu 
study of the linear delay differential equations and system with distributed delay (fundamental theory, stability, oscillation behavior, etc.) was done by A.D. Myshkis in his fundamental monograph [16]. The oscillation theory for neutral equations and systems (including the problems of stability, existing of non-oscillatory and bounded non-oscillatory solutions) is generally speaking more difficult. As an important branch of the applied theory of differential equations every year, during the last decades many papers on theoretical and applied aspects ofthe oscillation theory are published [20, 17, 4, 11, 2, 7, 5].

For more details see e.g., the important books by Saker [18], Erbe, Kong and Zhang [9], Agarwal, Berezansky, Braverman, Domoshnitsky [1], Bainov, Mishev [3], Gyori, Ladas[10] and the references therein.

The aim of this paper is to extend and improve the results obtained in [20] in the particular case of one constant delay in the neutral part of the considered system, for the general case of distributed delay in the neutral part. Moreover the additional conditions introduced by us for the general case are not additional restrictions for the case considered in [20].

\section{Preliminaries}

We consider the following linear delayed system of neutral type with distributed delay

$$
\frac{\mathrm{d}}{\mathrm{d} t}\left(x(t)+\delta \int_{-\tau}^{0} \mathrm{~d} v(s) x(t+s)\right)_{-\sigma}^{0} \mathrm{~d} u(s) x(t+s)=0
$$

where $\delta \in\{-1,1\}, \sigma>0, \tau>0, x:[0, \infty) \rightarrow R^{n}, v:[-\tau, 0] \rightarrow R L^{n}, u:$ $[-\sigma, 0] \rightarrow R L^{n}$. By $R L^{n}$ we denote the linear space of the $n \times n$ matrices $A=\left\{a_{i j}\right\}_{i, j=1}^{n}, a_{i j} \in R^{1}, n \geq 1$ is integer.

Definition 1. For each $a, b \in R^{1}, a<b$ we will denote by $B V[a, b]$ the linear space of matrix valued functions $v:[a, b] \rightarrow R L^{n}$ with bounded variation on $[a, b]$.

We suppose that for the functions $v:[-\tau, 0] \rightarrow R L^{n}$ and $u:[-\sigma, 0] \rightarrow R L^{n}$ the following conditions (S) are fulfilled:

(S1) The function $v \in B V[-\tau, 0]$ is left-side continuous on $[-\tau, 0]$, $\lim _{t \rightarrow+0}\left[\operatorname{Var}_{s \in[-t, 0]}(v(s))\right]=v(0)=0$ and $\operatorname{det}(v(-\tau+0)-v(-\tau-0)) \neq 0$.

(S2) The function $u \in B V[-\sigma, 0]$ is left-side continuous on $[-\sigma, 0)$ and $\operatorname{det}(u(-\sigma+0)-u(-\sigma-0)) \neq 0$. 
Remark 1. The condition (S1) means that the function $v \in B V[-\tau, 0]$ is atomic at $s=-\tau$ and non-atomic at $s=0$. For the function $u \in B V[-\sigma, 0]$ the condition (S2) means that it is atomic at $s=-\sigma$ and for $s=0$ the function can be atomic as well as non-atomic.

Let $A=\left\{a_{i j}\right\} \in R L^{n}$ is an arbitrary matrix, and $x \in R^{n}$ is an arbitrary vector-column. We will denote with $A^{T}=\left\{a_{j i}\right\}$ the transposed matrix and with $x^{T}=\left(x_{1}, \ldots x_{n}\right)$ the transposed vector of $x$. We introduce the notations

$$
\begin{gathered}
\|x\|_{k}=\left(\sum_{i=1}^{n}\left|x_{i}\right|^{k}\right)^{\frac{1}{k}}, 1 \leq k<\infty, \quad\|x\|_{\infty}=\max _{1 \leq i \leq n}\left\{\left|x_{i}\right|\right\}, \\
\|A\|_{k}=\sup _{x \in R^{n}, x \neq 0} \frac{\|A x\|_{k}}{\|x\|_{k}}, k=1,2, \ldots, \infty
\end{gathered}
$$

Definition 2. (see [8]) The function $\mu_{k}: R L^{n} \rightarrow R, k=1,2, \ldots, \infty$ defined with the equality

$$
\mu_{k}(A)=\lim _{\epsilon \rightarrow+0} \frac{\|I+\epsilon A\|_{k}-1}{\epsilon}, A \in R L^{n}, k=1,2, \ldots, \infty
$$

where $I \in R L^{n}$ is the unit matrix, is called logarithmic norm (Lozinskii measure).

Let us denote with $S p(A)$ the spectrum of $A$ and let us denote with $S(A):=$ $\sup \{\operatorname{Re} \lambda \mid \lambda \in S p(A)\}$ the spectral bound of $A$. The logarithmic norm (Lozinskii measure) is not a norm (measure) in common sense, because it can take negative values too. It is well known that for every square matrix $A$ the logarithmic norm of $A$ is related to his numerical bound (see [15]). Below we present some basic properties of the logarithmic norm for square matrices.

Lemma 1. (see $[19,6])$ Let $A, B \in R L^{n}, k=1,2, \ldots, \infty, \alpha \geq 0, \beta \in R^{1}$. Then the following relations hold:

i. $\mu_{k}(\alpha A+\beta I)=\alpha \mu_{k}(A)+\beta \quad ; \quad \mu_{k}(A)+\mu_{k}(-A) \geq 0 ;$

ii. $-\|A\|_{k} \leq-\mu_{k}(-A) \leq \mu_{k}(A) \leq\|A\|_{k} ; \quad\left|\mu_{k}(A)-\mu_{k}(B)\right| \leq\|A-B\|_{k}$;

iii. $\mu_{k}(-A) \leq \operatorname{Re} \lambda \leq \mu_{k}(A)$ for each $\lambda \in S p(A)$;

iv. $\mu_{1}(A)=\sup _{1 \leq j \leq n}\left\{a_{j j}+\sum_{i=1, i \neq j}^{n}\left|a_{i j}\right|\right\}, \quad \mu_{2}(A)=\frac{1}{2} S\left(A+A^{T}\right)$,

$$
\mu_{\infty}(A)=\sup _{1 \leq i \leq n}\left\{a_{i i}+\sum_{j=1, j \neq i}^{n}\left|a_{i j}\right|\right\} .
$$


Below in general we denote by $\mu(A)$ any one of $\mu_{k}(A), k=1,2, \ldots, \infty$.

Remark 2. It is clear that from Lemma 1 it follows that the logarithmic norm $\mu_{k}: L R^{n} \rightarrow R^{1}$ is a continuous function, if the topology in $R L^{n}$ is induced by the norm $\|\cdot\|_{k}, k=1,2, \ldots, \infty$. Moreover if $v \in B V[a, b]$, then the function $\mu \circ v:[a, b] \rightarrow R^{1}$ has bounded variation on $[a, b]$ too.

Let $t^{*}=\max (\tau, \sigma)$ and denote with $C\left(\left[-t^{*}, 0\right], R^{n}\right)$ the linear space of the continuous functions $\varphi:\left[-t^{*}, 0\right] \rightarrow R^{n}$ and with $C^{1}\left(\left[t_{0},+\infty\right), R^{n}\right), t_{0} \in$ $\left[-t^{*},+\infty\right)$ the linear space of the continuous differentiable functions $x:\left[t_{0},+\infty\right) \rightarrow$ $R^{n}$.

Definition 3. The function $x \in C\left(\left[t^{*},+\infty\right), R^{n}\right)$ is called a solution of the system (1) if $x(t)+\delta \int_{-\tau}^{0} \mathrm{~d} v(s) x(t+s) \in C^{1}\left([0,+\infty), R^{n}\right), x(t)$ satisfies the system (1) for each $t \geq 0$ and $x(t)=\varphi(t), t \in\left[-t^{*}, 0\right]$ for some initial function $\varphi \in C\left(\left[-t^{*}, 0\right], R^{n}\right)$.

Definition 4. The solution $x(t)=\left(x_{1}(t), \ldots, x_{n}(t)\right)^{T}$ of $(1)$ is said to be oscillatory (strongly oscillatory) if there exists an index $i, 1 \leq i \leq n$ and there exists a sequence $\left\{t_{k}^{i}\right\}_{k=1}^{+\infty}, \lim _{k \rightarrow+\infty} t_{k}^{i}=+\infty$ such that $x_{i}\left(t_{k}^{i}\right)=0\left(x_{i}\left(t_{k}^{i}\right) x_{i}\left(t_{k+1}^{i}\right)<\right.$ 0 ) for each $k \geq 1$. If all solutions of system (1) are oscillatory we call the system (1) oscillatory.

Definition 5. The solution $x(t)=\left(x_{1}(t), \ldots, x_{n}(t)\right)^{T}$ of $(1)$ is said to be non-oscillatory, if for each index $i, 1 \leq i \leq n$, there exists a point $t_{i} \geq 0$ such that $\left|x_{i}(t)\right|>0$ for each $t \geq t_{i}$.

Definition 6. The function $w \in B V[a, b]$ will be called monotone increasing (decreasing) on $[a, b]$ with respect to the logarithmic norm $\mu$, if for each numbers $c, d \in[a, b], c<d(c>d)$ the inequality $\mu(w(c)-w(d)) \leq 0$ is fulfilled. If this inequality is strictly then we call the function strictly increasing (decreasing) on $[a, b]$ with respect to the logarithmic norm $\mu$.

Let define the function $F: R^{1} \rightarrow R L^{n}$ by the following equality

$$
F(\lambda)=\lambda I+\lambda \delta \int_{-\tau}^{0} e^{\lambda s} \mathrm{~d} v(s)+\int_{-\sigma}^{0} e^{\lambda s} \mathrm{~d} u(s)
$$

Obviously the function $F(\lambda)$ is continuous for $\lambda \in R^{1}$ if the topology in $R L^{n}$ is induced by the norm $\|.\|_{k}, k=1,2, \ldots,+\infty$. Moreover the function $\mu \circ F: R^{1} \rightarrow R^{1}$ is continuous for $\lambda \in R^{1}$ too.

The next simple but important lemma is well known. 
Lemma 2. The necessary and sufficient condition for existing of a nonoscillatory solution of the system (1) of the type $x(t)=e^{\lambda t} C(\lambda)$, where $\lambda \in$ $R^{1}, C(\lambda) \in R^{n}$, is the characteristic equation

$$
\operatorname{det}\left(\lambda\left(I+\delta \int_{-\tau}^{0} e^{\lambda s} \mathrm{~d} v(s)\right)+\int_{-\sigma}^{0} e^{\lambda s} \mathrm{~d} u(s)\right)=0
$$

to have at least one real root.

Corollary 1. The necessary condition the system (1) to be oscillating is the characteristic equation (3) to have no real roots.

\section{Main Results}

In this section we present three theorems giving explicit and easy computable sufficient conditions for existing of bounded and unbounded non-oscillatory solutions of (1). We note that the results are proved for the case that the functions $u \in B V[-\sigma, 0]$ and $v \in B V[-\tau, 0]$ are non-monotonic with respect to the logarithmic norm as in [20].

For each function $w=\left\{w_{i j}\right\} \in B V\left[-t^{*}, 0\right], n \geq 1$ we denote by $J_{w_{i j}}[a, b]$ the set of jumps of the function $w_{i j}:\left[-t^{*}, 0\right] \rightarrow R^{1}$, i.e.

$$
J_{w_{i j}}[a, b]=\left\{t \in[a, b]|| w_{i j}(t+0)-w_{i j}(t-0) \mid>0\right\} .
$$

By $J_{w}[a, b]$ we will denote the set $J_{w}[a, b]=\bigcup_{i, j=1}^{n} J_{w_{i j}}[a, b]$ and by $E_{w}[a, b]$ the set $E_{w}[a, b]=[a, b] \backslash J_{w}[a, b]$.

Lemma 3. Let the following conditions are fulfilled:

1. The function $w \in B V\left[-t^{*}, 0\right]$.

2. The function $f:\left[-t^{*}, 0\right] \rightarrow R^{1}$ is monotonous and has a constant sign on $\left[-t^{*}, 0\right]$.

3. $J_{w}\left[-t^{*}, 0\right] \cap J_{f}\left[-t^{*}, 0\right]=\emptyset$.

Then the following inequalities hold:

a) If $f$ is decreasing and positive (negative), then

$$
\mu\left(\int_{-t^{*}}^{0} f(s) \mathrm{d} w(s)\right) \leq \int_{-t^{*}}^{0} f(s) \mathrm{d} \mu\left(w(s)-w\left(-t^{*}\right)\right)
$$




$$
\left(\mu\left(\int_{-t^{*}}^{0} f(s) \mathrm{d} w(s)\right) \leq \int_{-t^{*}}^{0} f(s) \mathrm{d} \mu(w(s)-w(0))\right)
$$

b) If $f$ is increasing and positive (negative), then

$$
\begin{gathered}
\mu\left(\int_{-t^{*}}^{0} f(s) \mathrm{d} w(s)\right) \leq-\int_{-t^{*}}^{0} f(s) \mathrm{d} \mu(w(0)-w(s)) \\
\left(\mu\left(\int_{-t^{*}}^{0} f(s) \mathrm{d} w(s)\right) \leq-\int_{-t^{*}}^{0} f(s) \mathrm{d} \mu\left(w\left(-t^{*}\right)-w(s)\right)\right.
\end{gathered}
$$

Proof. Since the proof of all inequalities is very similar we will proof only the first of them. Let $f$ is decreasing and positive. Then it follows that

$$
\begin{aligned}
& \mu\left(\int_{-t^{*}}^{0} f(s) \mathrm{d} w(s)\right)=\mu\left(\int_{-t^{*}}^{0} f(s) \mathrm{d}\left(w(s)-w\left(-t^{*}\right)\right)=\right. \\
& \quad=\mu\left(f(0)\left(w(0)-w\left(-t^{*}\right)\right)-\int_{-t^{*}}^{0}\left(w(s)-w\left(-t^{*}\right)\right) \mathrm{d} f(s)\right) \leq \\
& \leq f(0) \mu\left(w(0)-w\left(-t^{*}\right)\right)+\mu\left(\int_{-t^{*}}^{0}\left(w(s)-w\left(-t^{*}\right)\right) \mathrm{d}(-f(s))\right) \leq \\
& \quad \leq f(0) \mu\left(w(0)-w\left(-t^{*}\right)\right)+\int_{-t^{*}}^{0} \mu\left(w(s)-w\left(-t^{*}\right)\right) \mathrm{d}(-f(s)) \leq \\
& \leq \int_{-t^{*}}^{0} f(s) \mathrm{d} \mu\left(w(s)-w\left(-t^{*}\right)\right)
\end{aligned}
$$

It is simply to see that from the conditions of the lemma it follows that all Styltes integrals exist and the integration of the parts is correct.

Remark 3. The assertions of Lemma 3 have been proved as Lemma 2.2 in the remarkable work [12] in the case of monotonic and continuous functions $f(x)$. The introduced by us condition 3 of Lemma 3 permits to generalize the assertion to the case when the function $f(x)$ is monotonic only. Since the 
condition 3 of Lemma 3 is evidently fulfilled in the case when the function $f(x)$ is monotonic and continuous, then this condition is not an additional restriction for this case.

Remark 4. It is simply to see that $\lambda=0$ is a root of the equation (4) if and only if, when $\operatorname{det}(u(-\sigma)-u(0))=0$ and then without any additional conditions, the system (1) have a bounded non-oscillatory solution. Then from the condition $\operatorname{det}(u(-\sigma)-u(0)) \neq 0$ used below it follows only, that $\lambda=0$ is not a root of the equation (4).

Theorem 1. Let the following conditions are fulfilled: odd.

1. The conditions (S) hold, $\operatorname{det}(u(0)-u(-\sigma))<0$ and the number $n$ is

2. $\delta=-1$ and $\sup _{s \in E_{v}[-\tau, 0]} \mu(-v(s))<1$

Then the system (1) has at least one unbounded non-oscillatory solution.

Proof. Since the function $\operatorname{det} F(\lambda)$ is continuous for $\lambda \in R^{1}$, it is enough to prove that there exist two numbers $\lambda_{1}, \lambda_{2} \in[0,+\infty), \lambda_{1} \neq \lambda_{2}$, such that $\operatorname{det}\left(F\left(\lambda_{1}\right)\right) \operatorname{det}\left(F\left(\lambda_{2}\right)\right) \leq 0$. Since the number $n$ is odd, then according to Lemma 2 and Gerschgorin's theorem this inequality will be true, if $\mu\left(F\left(\lambda_{1}\right)\right) \leq 0$ and $\mu\left(-F\left(\lambda_{2}\right)\right) \leq 0$.

From condition 1 of the theorem it follows that $\operatorname{det}(F(0))<0$ and then we can choise $\lambda_{1}=0$. In virtue of Lemma 1 for each $\lambda>0$ from (2) it follows the estimation

$$
\begin{aligned}
\mu(-F(\lambda)) & =\mu\left(-\lambda I+\lambda \int_{-\tau}^{0} e^{\lambda s} \mathrm{~d} v(s)-\int_{-\sigma}^{0} e^{\lambda s} \mathrm{~d} u(s)\right) \leq \\
& \leq-\lambda+\lambda \mu\left(\int_{-\tau}^{0} e^{\lambda s} \mathrm{~d} v(s)\right)+\mu\left(-\int_{-\sigma}^{0} e^{\lambda s} \mathrm{~d} u(s)\right) .
\end{aligned}
$$

Applying Lemma 3, from (4) it follows that

$$
\mu(-F(\lambda)) \leq-\lambda-\lambda \int_{-\tau}^{0} e^{\lambda s} \mathrm{~d} \mu(v(0)-v(s))-\int_{-\sigma}^{0} e^{\lambda s} \mathrm{~d} \mu(u(s)-u(0)) .
$$

Since $v \in B V[-\tau, 0]$ and therefore it has countable many bounded jumps, then for the second addend on the right side of (5) after integration of parts we 
receive the estimation

$$
\begin{aligned}
& -\lambda \int_{-\tau}^{0} e^{\lambda s} \mathrm{~d} \mu(v(0)-v(s)) \leq \\
& \quad \leq \lambda e^{-\lambda \tau}\left(\mu(v(0)-v(-\tau))-\sup _{s \in E_{v}[-\tau, 0]} \mu(v(0)-v(s))\right)+ \\
& \quad+\lambda \sup _{s \in E_{v}[-\tau, 0]} \mu(v(0)-v(s)) .
\end{aligned}
$$

For the third addend on the right side of (5) similar way we obtain

$$
\begin{aligned}
& -\int_{-\sigma}^{0} e^{\lambda s} \mathrm{~d} \mu(u(s)-u(0)) \leq \\
& \quad \leq e^{-\lambda \sigma}\left(\mu(u(-\sigma)-u(0))-\sup _{s \in[-\sigma, 0]} \mu(u(s)-u(0))\right)+ \\
& \quad+\sup _{s \in[-\sigma, 0]} \mu(u(s)-u(0)) .
\end{aligned}
$$

Then taking into account (5), (6) and (7), from condition 2 of the theorem follows that $\mu(-F(\lambda)) \leq 0$, if $\lambda>0$ is sufficiently large. Therefore, from Lemma 2 it follows that the system (1) has at least one unbounded non-oscillatory solution.

Theorem 2. Let the following conditions are fulfilled:

1. The conditions $(S)$ hold, $\operatorname{det}(u(0)-u(-\sigma))<0$ and the number $n$ is odd.

$$
\text { 2. } \delta=1 \text { and } \sup _{s \in E_{v}[-\tau, 0]} \mu(v(s))<1
$$

Then the system (1) has at least one unbounded non-oscillatory solution.

Proof. As in the proof of Theorem 1 we can conclude that $\operatorname{det}(F(0))<0$. In similar way as in Theorem 1 using Lemma 1 and Lemma 3 we obtain that the following inequality

$$
\mu(-F(\lambda)) \leq-\lambda-\lambda \int_{-\tau}^{0} e^{\lambda s} \mathrm{~d} \mu(v(s)-v(0))-\int_{-\sigma}^{0} e^{\lambda s} \mathrm{~d} \mu(u(s)-u(0))
$$


is true for each $\lambda>0$. The difference between inequalities (5) and (8) is the second addend on the right side. For the second addend on the right side of (8) we have the following estimation

$$
\begin{aligned}
& -\lambda \int_{-\tau}^{0} e^{\lambda s} \mathrm{~d} \mu(v(s)-v(0)) \leq \\
& \quad \leq \lambda e^{-\lambda \tau}\left(\left(\mu(v(-\tau)-v(0))-\sup _{s \in E_{v}[-\tau, 0]} \mu(v(s)-v(0))\right)+\right. \\
& \quad+\lambda \sup _{s \in E_{v}[-\tau, 0]} \mu(v(s)-v(0)) .
\end{aligned}
$$

From condition 2 of Theorem 2 and from (7), (8) and (9) it follows that $\mu(-F(\lambda)) \leq 0$, if $\lambda>0$ is sufficiently large. Thus the equation (3) has at least one real positive root.

Corollary 2. Let the following conditions are fulfilled:

1. The conditions (S) hold and the number $n$ is odd.

2. $\mu(u(0)-u(-\sigma)) \leq 0$ and $\operatorname{det}(u(-\sigma)-u(0)) \neq 0$.

3. One of the following two conditions holds:

$$
\begin{aligned}
& \text { 3.1. } \delta=-1 \text { and } \sup _{s \in E_{v}[-\tau, 0]} \mu(-v(s))<1 . \\
& \text { 3.2. } \delta=1 \text { and } \sup _{s \in E_{v}[-\tau, 0]} \mu(v(s))<1 .
\end{aligned}
$$

Then the system (1) has at least one unbounded non-oscillatory solution.

Proof. Condition 2 implies that $\operatorname{det}(u(0)-u(-\sigma))<0$ and therefore the assertion of the Corollary 2 follows immediately from Theorem 1 or Theorem 2 .

Remark 5. Generally speaking from the condition $\mu(u(0)-u(-\sigma))<0$ it does not follow that the function $u(s)$ is monotonous on $[-\sigma, 0]$ with respect to the logarithmic norm.

Remark 6. In [20] the results of Theorems 1 and 2 are obtained in the particular case when $\delta=1$ and $v(s)=H(-(s+\tau)) A, A \in R L^{n}$, where $H(s)$ is the Heaviside function, under two additional restrictive conditions - the function $u(s)$ must be nonatomic at zero and monotonic with respect to the logarithmic norm. Obviously $E_{v}[-\tau, 0]=(-\tau, 0]$ and for this function the condition 3 of Theorem 2 holds. Since for each $s \in E_{v}[-\tau, 0]=(-\tau, 0]$ the function $-v(s)=$ 
$-H(-(s+\tau)) A=0$, then for the function $v(s)=H(-(s+\tau)) A, A \in R L^{n}$ the condition 3 of Theorem 1 holds also. Since the introduced by us conditions 3 of Theorems 1 and 2 are evidently fulfilled in the case considered in [20], then these conditions are not additional restrictions for this case.

For some applications it is important to know how the relation between $\tau$ and $\sigma$ influences on the existence of non-oscillatory solutions for the system (1) and their asymptotical behavior.

Theorem 3. Let the following conditions are fulfilled:

1. The conditions $(S)$ hold, $\operatorname{det}(u(0)-u(-\sigma))<0$ and the number $n$ is odd.

2. One of the following two conditions holds:

2.1. $\delta=-1$ and $\sup _{s \in E_{v}[-\tau, 0]} \mu(v(-\tau)-v(s))<0$.

2.2. $\delta=1$ and $\sup _{s \in E_{v}[-\tau, 0]} \mu(v(s)-v(-\tau))<0$.

3. $\tau>\sigma$.

Then the system (1) has at least one unbounded non-oscillatory solution $x(t)=\left(x_{1}(t), \ldots, x_{n}(t)\right)^{T}$ such that $\lim _{t \rightarrow+\infty} x_{i}(t)=0$ for each $i, 1 \leq i \leq n$.

Proof. Let suppose that $\operatorname{det}(F(\lambda)) \neq 0$ for each $\lambda \in(-\infty, 0]$. Since $\operatorname{det}(F(\lambda))$ is continuous function for $\lambda \in R^{1}$ and from condition 1 it follows that $\operatorname{det}(F(0))<$ 0 and therefore $\operatorname{det}(F(\lambda))<0$ for each $\lambda \in(-\infty, 0]$. This implies that the equation $\operatorname{det}(\xi I-F(\lambda))=0$ for each $\lambda \leq 0$ has at least one real negative root $\xi(\lambda)$, i.e. the matrix $F(\lambda)$ has at least one real negative eigenvalue $\xi(\lambda)$. Then from Lemma 1 it follows that for each $\lambda \leq 0$ the inequality $\mu(-F(\lambda))>0$ holds.

Let $\delta=-1$. In virtue of Lemma 1 and Lemma 3 for each $\lambda \leq 0$ we obtain the estimation

$$
\mu(-F(\lambda)) \leq-\lambda-\lambda \int_{-\tau}^{0} e^{\lambda s} \mathrm{~d} \mu(v(-\tau)-v(s))+\int_{-\sigma}^{0} e^{\lambda s} \mathrm{~d} \mu(u(-\sigma)-u(s))
$$


For the third addend on the right side of (10) we obtain

$$
\begin{aligned}
& \int_{-\sigma}^{0} e^{\lambda s} \mathrm{~d} \mu(u(-\sigma)-u(s)) \leq \\
& \quad \leq \mu(u(-\sigma)-u(0))-\sup _{s \in[-\sigma, 0]} \mu(u(-\sigma)-u(s))+ \\
& \quad+\sup _{s \in[-\sigma, 0]} \mu(u(-\sigma)-u(s)) e^{-\lambda \sigma} .
\end{aligned}
$$

Since $\mu(u(-\sigma)-u(0))>0$, then $\sup _{s \in[-\sigma, 0]} \mu(u(-\sigma)-u(s))>0$ also. Taking into consideration that the function $v \in B V[-\tau, 0]$ and therefore it has countable many bounded jumps, then for the second addend on the right side of (10) after integration of parts we receive the estimation

$$
\begin{aligned}
& -\lambda \int_{-\tau}^{0} e^{\lambda s} \mathrm{~d} \mu(v(-\tau)-v(s)) \leq \\
& \quad \leq-\lambda \mu(v(-\tau)-v(0))+\lambda \sup _{s \in E_{v}[-\tau, 0]} \mu(v(-\tau)-v(s))- \\
& \quad-\lambda e^{-\lambda \tau} \sup _{s \in E_{v}[-\tau, 0]} \mu(v(-\tau)-v(s)) .
\end{aligned}
$$

From (10), (11), (12), conditions 2 and 3 follows that $\mu(-F(\lambda)) \leq 0$ if $|\lambda|$ is sufficiently large, which is a contradiction.

If $\delta=1$, then similarly as in the case $\delta=-1$, we receive the following inequality

$$
\mu(-F(\lambda)) \leq-\lambda-\lambda \int_{-\tau}^{0} e^{\lambda s} \mathrm{~d} \mu(v(s)-v(-\tau))+\int_{-\sigma}^{0} e^{\lambda s} \mathrm{~d} \mu(u(-\sigma)-u(s)) .
$$

For the third addend on the right side of (13) the estimation (11) holds, and for the second addend we have the following estimation

$$
\begin{aligned}
& -\lambda \int_{-\tau}^{0} e^{\lambda s} \mathrm{~d} \mu(v(s)-v(-\tau)) \leq \\
& \quad \leq-\lambda \mu(v(0)-v(-\tau))+\lambda \sup _{s \in E_{v}[-\tau, 0]} \mu(v(s)-v(-\tau))- \\
& \quad-\lambda e^{-\lambda \tau} \sup _{s \in E_{v}[-\tau, 0]} \mu(v(s)-v(-\tau)) .
\end{aligned}
$$


Then from (11), (13), (14), condition 2 and 3 follows that $\mu(-F(\lambda)) \leq 0$ if $|\lambda|$ is sufficiently large, which is a contradiction. Thus the equation (3) has at least one real negative root.

Example 1. Let in the system (1) $\delta=-1$ and $\tau>0$. Consider the function $v(s)$ in the form

$$
v(s)=\sum_{k=1}^{m} H_{k}\left(-\left(s+\tau_{k}\right)\right) A_{k},
$$

where $A_{k} \in R L^{n}, \tau_{k} \in(0, \tau], k=1,2, \ldots, m, 0<\tau_{1}<\tau_{2}<\ldots<\tau_{m}=\tau$. Let the relation $\sup _{k} \mu\left(\sum_{i=k}^{m}\left(A_{i}\right)\right)<0, k=1,2, \ldots, m$ holds. Since $E_{v}[-\tau, 0]=$ $\left(-\tau_{1}, 0\right] \cup\left(\bigcup_{i=1}^{m-1}\left(-\tau_{i+1},-\tau_{i}\right)\right)$, then by means of Lemma 1 it is not difficult to see, that for the function $v(s)$ defined by (15) the condition 2.1 of Theorem 3 holds. Obviously this relation is fulfilled if $\mu\left(A_{k}\right) \leq 0, k=1,2, \ldots, m$.

\section{Discussion}

Since the matrices $A \in R L^{n}$ with dimension $n=1$ are real numbers, therefore formally $\mu(A)=\operatorname{det} A$. Then the two cases mentioned in conditions 2 of Theorem 3 are being identical. However for $A \in R L^{n}$ when $n \geq 3$ generally speaking $-\mu(A) \neq \mu(-A)$ and then we can see that the two cases mentioned in conditions 2 of Theorem 3 are different. The appearing of these cases is the effect of the high dimension.

The next examples illustrate the Theorems, proved above.

Example 2. Let consider the system (1), when $n=3, \delta=-1, A, B_{0}, B_{1} \in$ $R L^{3}$ and let consider the functions $v(s), u(s)$ in the form

$$
v(s)=-H(-(s+2)) A, u(s)=H(-(s+3)) B_{1}+H(s) B_{0} .
$$

If we choose the matrices as follow:

$$
A=\left(\begin{array}{ccl}
0.5 & 0 & 0 \\
0 & 1 & 0 \\
0 & 0 & 2
\end{array}\right), \quad B_{0}=\left(\begin{array}{ccc}
-5 & 0 & 0 \\
0 & -5 & 0 \\
0 & 0 & -5
\end{array}\right), \quad B_{1}=\left(\begin{array}{lll}
4 & 0 & 0 \\
0 & 3 & 0 \\
0 & 0 & 2
\end{array}\right),
$$

then all condition of Theorem 1 are fulfilled, and the characteristic equation (3) will have the form

$$
\operatorname{det}\left(\lambda\left(I-A e^{-2 \lambda}\right)+\left(B_{0}-B_{1} e^{-3 \lambda}\right)\right)=0
$$




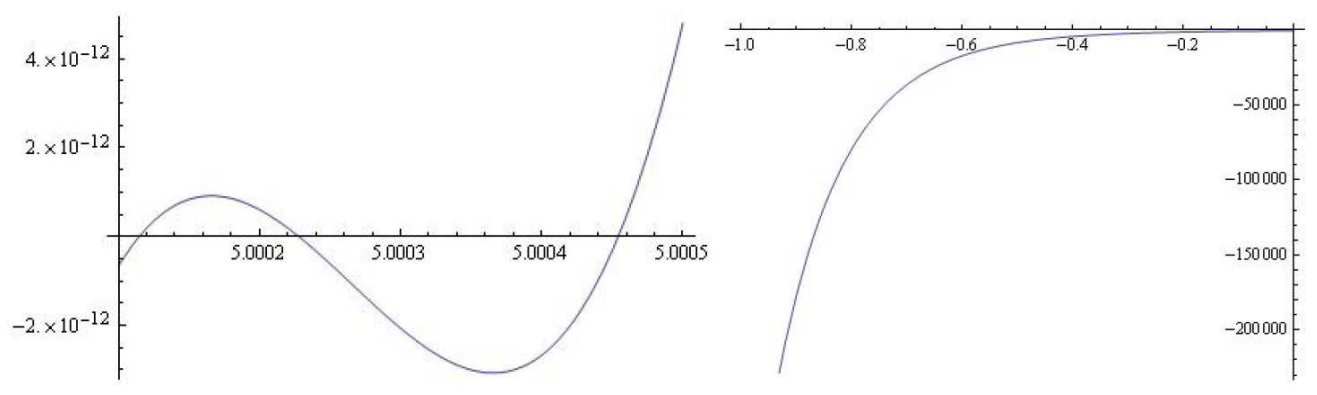

Figure 1

Calculating for example with Wolfram Mathematica we receive that the characteristic equation (16) has three real positive roots

$$
s_{1}=5.00011, s_{2}=5.00023, s_{3}=5.00045
$$

as shown in the left graphic in Figure 1.

Thus the system (1) has at least one unbounded non-oscillatory solution.

Moreover, Example 2 illustrate that in the case $\delta=-1$ Condition 3 of Theorem 3 is essential for its validity.

It is simple to see, that $v(-2)=-A, v(s)=0, s \in E_{v}[-\tau, 0]$ and therefore Conditions 1 and 2.1 of Theorem 3 are fulfilled. Only the Condition 3 is not fulfilled and because of that, the characteristic equation (16) has no negative real roots (as shown in the right graphic in Figure 1), i.e. the system (1) has no bounded positive solutions .

The next example illustrate, that Condition 3 of Theorem 3 is essential in the case $\delta=1$ too.

Example 3. Let consider the system (1), when $n=3, \delta=1$, and let consider the functions $v(s), u(s)$ in the form

$$
v(s)=H(-(s+2)) A, u(s)=H(-(s+3)) B_{1}+H(s) B_{0} .
$$

We choose the same matrices $A, B_{0}, B_{1} \in R L^{3}$ as in Example 2 .

The conditions 1 and 2.2 of Theorem 3 are obviously fulfilled, but the condition 3 is not. The characteristic equation (3) will have the form

$$
\operatorname{det}\left(\lambda\left(I+A e^{-2 \lambda}\right)+\left(B_{0}-B_{1} e^{-3 \lambda}\right)\right)=0
$$

Calculating with Wolfram Mathematica we receive that the characteristic equation (17) has an unic real root $s=4.99989$ and hence the system (1) has no bounded positive solutions (as shown in Figure 2). 

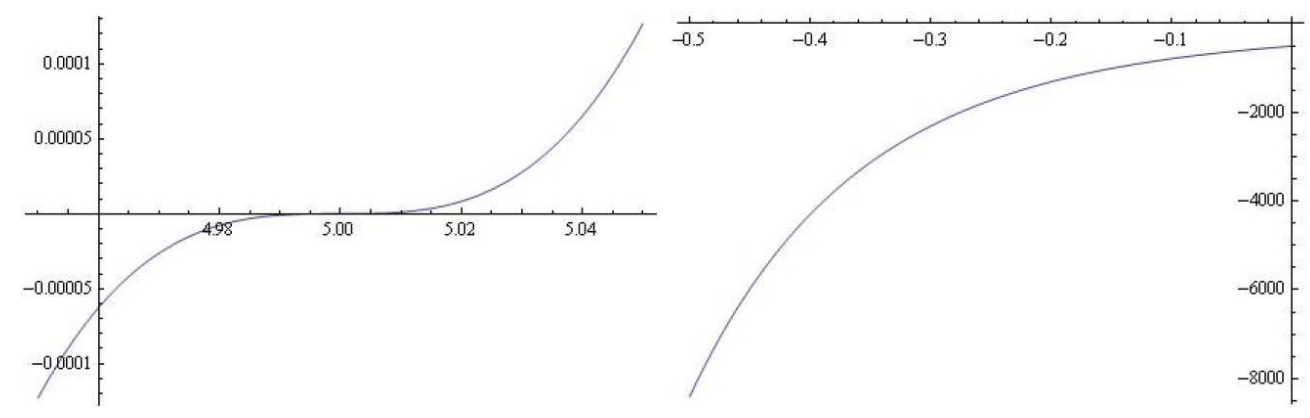

Figure 2

Both examples illustrate, that Condition 3 of Theorem 3 is essential and is not determined from the technic, used in the proof of Theorem 3.

\section{References}

[1] R. Agarwal, L. Berezansky, E. Braverman, A. Domoshnitsky, Nonoscillation Theory of Functional Differential Equations with Applications, New York, USA, Springer-Verlag (2012), doi: 10.1007/978-1-4614-3455-9.

[2] V. Angelov, D. Angelova, Oscillatory Solutions of Neutral Equations with Polynomial Nonlinearities, International Journal of Differential Equations (2011), 1-12, doi: 10.1155/2011/949547.

[3] D. Bainov, D. Mishev, Oscillations Theory for Neutral Delay Differential Equations with Delay, Adam Hilger Ltd, Bristol (1991).

[4] Basak Karpuz, Ozkan Okalan, Mustafa Kemal Yildiz, Oscillation of higherorder nonlinear delay differential equations with oscillatory coefficients, Turk. J. Math., 33 (2009), 259-263, doi: 10.3906/mat-0704-2.

[5] L. Berezansky, E. Braverman, L. Idels, The Mackey-Glass model of respiratory dynamics: Review and results, Nonlinear Analysis, 75 (2012), 6043-6052, doi: 10.1016/j.na.2012.06.013.

[6] F.F. Bonsall, J. Duncan, Numerical Ranges of Operators on Normed Spaces and Elements of Normed Algebras, London, Cambridge University Press (1971). 
[7] T. Candan, The existence of nonoscillatory solutions of higher order nonlinear neutral equations, Applied Mathematics Letters, 25 (2012), 412-416, doi: 10.1016/j.aml.2011.09.025.

[8] G. Dahlquist, Stability and Error Bounds in the Numerical Integration of Ordinary Differential Equations, Uppsala, Almqvist \& Wiksell (1958).

[9] L.H. Erbe, Q. Kong, B.G. Zhang, Oscillation Theory for Functional Differential Equations, New York, Marcel Dekker (1995).

[10] I. Gyori, G. Ladas, Oscillation Theory of Delay Differential Equations With Applications, Oxford, Clarendon Press (1991).

[11] I. Ilavska, A. Najmanova, R. Olach, Oscillation of nonlinear neutral differential system, Journal of Applied Mathematics, 3, No. 2 (2010), 107-112.

[12] J. Kirchner, U. Stroinski, Explicit oscillation criteria for systems of neutral differential equations with distributed delay, Tuebinger Berichte zur Funktional Analysis (1994), 1-19.

[13] V. Kolmanovskii, A. Mishkis, Introduction to the Theory and Applications of Functional Differential Equations, Mathematics and its Applications, Kluwer Academic Publishers, Dordreht (1999).

[14] V. Kolmanovskii, A. Mishkis, Applied Theory of Functional Differential Equations, Mathematics and its Applications, 85, Kluwer Academic Publishers, Dordreht (1992).

[15] G. Lumer, Semi-inner-product spaces, Trans. Amer. Math. Soc., 100 (1961), 29-43.

[16] A.D. Myshkis, Linear Differential Equations with Retarded Argument, Moscow, Nauka (1972), In Russian.

[17] Quanxin Zhang, Li Gao, Yuanhong Yu, Oscillation criteria for third-order neutral differential equations with continuously distributed delay, Applied Mathematics Letters, 25 (2012), 1514-1519, doi: 10.1016/j.aml.2012.01.007.

[18] S. Saker, Oscillation Theory of Delay Differential and Difference Equations Second and Third Orders, Saarbrucken, VDM Verlag Dr. Muller (2010).

[19] G. Soederlind, The logarithmic norm. History and modern theory, BIT Numerical Mathematics, 46, No. 3 (2006), 631-652. 
[20] Xin Zhou Yan, Non-oscillatory solutions of linear delay functional differential system of neutral type, Advanced Material Research Vols., 482-484 (2012), 66-69, doi: 10.4028/www.scientific.net/AMR.482-484.66. 